\title{
Santilli Autotopisms of Partial Groups
}

\author{
Raúl M. Falcón ${ }^{1}$, Juan Núñez ${ }^{2}$ \\ ${ }^{1}$ Department of Applied Mathematics I, University of Seville, Seville, Spain \\ ${ }^{2}$ Department of Geometry and Topology, University of Seville, Seville, Spain
}

Email address:

rafalgan@us.es (R. M. Falcón), jnvaldes@us.es (J. Núñez)

\section{To cite this article:}

Raúl M. Falcón, Juan Núñez. Santilli Autotopisms of Partial Groups. American Journal of Modern Physics. Special Issue: Issue I: Foundations of Hadronic Mathematics. Vol. 4, No. 5-1, 2015, pp. 47-51. doi: 10.11648/j.ajmp.s.2015040501.16

\begin{abstract}
This paper deals with those partial groups that contain a given Santilli isotopism in their autotopism group. A classification of these autotopisms is explicitly determined for partial groups of order $\mathrm{n} \leq 4$.
\end{abstract}

Keywords: Partial Group, Isotopism, Classification

\section{Introduction}

In 1942, Albert [1] introduced the concept of isotopy of algebras: Two algebras $\left(A_{1}, \cdot\right)$ and $\left(A_{2}, *\right)$ over a field $K$ are said to be isotopic if there exist three regular linear transformations $f, g$ and $h$ from $A_{1}$ to $A_{2}$ such that

$$
f(u) * g(v)=h(u \cdot v) \text {, for all } u, v \in A_{1} .
$$

The algebra $A_{2}$ is then said to be isotopic to the algebra $A_{1}$, or, equivalently, $A_{2}$ is an isotope of $A_{l}$. The triple $\theta=(f, g, h)$ is an isotopy or isotopism between both algebras $A_{1}$ and $A_{2}$. If $f$ $=g=h$, then this is indeed an isomorphism. If the elements of $A_{1}$ and $A_{2}$ coincide, then the isotopism $\theta$ is said to be principal if $h$ is the trivial transformation $I d$, that is, if $h(u)=I d(u)=u$, for all $u \in A_{l}$. In this case, the algebra $A_{2}$ is said to be a principal isotope of $A_{l}$. In his original paper, Albert proposed the question as to whether a principal isotope of a Lie algebra is Lie. In this regard, he proved that a principal isotope $A_{2}$ of a Lie algebra $A_{l}$ with respect to a principal isotopism $(f, g, I d)$ is a Lie algebra if and only if, for all $u, v, w \in A_{l}$, it is verified that

$$
f(u) \cdot g(v)=-f(v) \cdot g(u) .
$$

$f(f(u) \cdot g(v)) \cdot g(w)-f(f(u) \cdot g(w)) \cdot g(v)-f(u) \cdot g(f(v) \cdot g(w))=0$.

In 1944, Bruck [2] introduced the concept of isotopically simple algebra as a simple algebra such that all their isotopic algebras are simple. He proved in particular that the Lie algebra of order $n \cdot(n-1) / 2$, consisting of all skew-symmetric matrices over any subfield of the field of all reals, under the Lie product $[u, v]=u \cdot v-v \cdot u$, is isotopically simple. Further, the Lie algebra of order $n \cdot(n-1)$ consisting of all
skew-Hermitian matrices in any field $R(i)$ (where $R$ is a subfield of the reals and $i^{2}=-1$ ), under the multiplication $[u$, $v]=u \cdot v-v \cdot u$, is an isotopically simple algebra over $R$.

More recently, in 1978, Santilli [3] generalized the associative product $u \cdot v$ between Hermitian generators of the universal enveloping associative algebra by considering the new product

$$
u * v=u \cdot T \cdot v
$$

where $T$ is a positive-definite operator called the isotopic element, which can indeed depend on distinct factors

$$
T=T\left(x, x^{\prime}, x^{\prime}, \ldots, \mu, \tau\right)
$$

The product

$$
[u, v]=u * v-v * u
$$

preserves the Lie axioms and is called the Lie-isotopic product. The application to Lie's theory (enveloping algebras, Lie algebras and Lie groups) that emerges from this new product is the so-called Lie-Santilli isotheory (see [3, pp. 287-290 and 329-374] and also [4-9]).

In the development of the isotheory, Santilli extended the unit of the ground field to the generalized unit or isounit

$$
I=I\left(\mathrm{x}, \mathrm{x}^{\prime}, \mathrm{x}^{\prime}, \ldots, \mu, \tau\right)=T^{-1}
$$

He defined then the isonumbers

$$
u=u * I\left(x, x^{\prime}, x^{\prime}, \ldots, \mu, \tau\right), \text { for all } u \in A .
$$

and the isoproduct

$$
[u, v]=u * v-v * u
$$


This isoproduct constitutes the Lie product of an isomorphic Lie algebra of $A$ whenever the isounit $\hat{I}$ is constant. In any other case, this determines a generalization of the classical notion (2) of isotopism. In order to analyze this fact, the authors [10] reinterpreted in 2006 the dependence on distinct factors of the isounit $\hat{I}$ as a family of classical Bruck's isotopisms. This reinterpretation became clearer shortly after [11] once the attention was focused not on isotopisms of algebras, but on isotopisms of partial quasigroups.

The term quasigroup was introduced in 1937 by Haussmann and Ore [12] to denote a nonempty set $Q$ endowed with a product ', such that if any two of the three symbols $u, v$ and $w$ in the equation $u \cdot v=w$ are given as elements of $Q$, then the third is uniquely determined as an element of $Q$. Its order is the cardinality of the underlying set, that is, the number of elements of the quasigroup $Q$. This is said to be a loop if it contains a unit element, that is, there exists an element $e \in Q$ such that $e \cdot u=u \cdot e=u$ for all $u \in Q$. Every associative loop is indeed a group. The multiplication table of a quasigroup of order $n$ is a Latin square of order $n$, that is, an $n \times n$ array with elements chosen from a set of $n$ distinct symbols such that each symbol occurs precisely once in each row and each column (see Figure 1).

\begin{tabular}{|l|l|l|l|}
\hline 2 & 3 & 4 & 1 \\
\hline 3 & 4 & 1 & 2 \\
\hline 4 & 1 & 2 & 3 \\
\hline 1 & 2 & 3 & 4 \\
\hline
\end{tabular}

Figure 1. Latin square of order 4.

A partial Latin square of order $n$ is an $n \times n$ array with elements chosen from a set of $n$ distinct symbols such that each symbol occurs at most once in each row and each column (see Figure 2). It constitutes the multiplication table of a finite partial quasigroup $(Q, \cdot)$ of order $n$. Let $u, v \in Q$. The product $u \cdot v$ is then an element of $Q$ or it is undefined. This last case is denoted as $u \cdot v=\varnothing$. By abuse of notation, it is also considered that $u \cdot \varnothing=\varnothing \cdot u=\varnothing$, for all $u \in Q$ and hence, the partial quasigroup is associative if $(u \cdot v) \cdot w=u \cdot(v \cdot w)$, for all $u, v, w$ $\in Q$. It is a partial loop if there exists an element $e \in Q$ such that $e \cdot u=u \cdot e \in\{u, \varnothing\}$ for all $u \in Q$ and there does not exist an element $e^{\prime} \neq e$ such that $e^{\prime} \cdot u=u$ or $u \cdot e^{\prime}=u$. Every associative partial loop constitutes a partial group.

\begin{tabular}{|c|c|c|c|}
\hline 1 & & & \\
\hline & 2 & & 4 \\
\hline 3 & & & \\
\hline 4 & & 3 & \\
\hline
\end{tabular}

Figure 2. Partial Latin square of order 4.

In 1943-44, Albert [13, 14] together with Bruck [15] extended the definition of isotopy from algebras to quasigroups. Particularly, two quasigroups $\left(Q_{1}, \cdot\right)$ and $\left(Q_{2}, *\right)$ of the same order are said to be isotopic if there exist three bijections $f, g$ and $h$ between their sets of elements such that

$$
f(u) * g(v)=h(u \cdot v), \text { for all } u, v \in Q_{l} .
$$

The definition can be naturally extended to partial quasigroups once it is considered $h(\varnothing)=\varnothing$. The triple $\theta=(f$, $g, h)$ is said to be an isotopism between $Q_{1}$ and $Q_{2}$ and it is denoted $Q_{2}=Q_{1}{ }^{\theta}$. If $Q_{2}=Q_{1}$, then the isotopism $\theta$ is said to be an autotopism of $Q_{l}$ and $f, g$ and $h$ are permutations of the elements of $Q_{l}$. The set of autotopisms of a (partial) quasigroup constitutes, therefore, a group with the component-wise composition of permutations.

In 2007, the authors [11] introduced the concept of Santilli isotopism between partial quasigroups. Specifically, an isotopism $\theta=(f, g, h)$ between two partial quasigroups $\left(Q_{l}, \cdot\right)$ and $\left(Q_{2}, *\right)$ is said to be a Santilli isotopism if there exist three elements $i_{f}, i_{g}$ and $i_{h}$ in $Q_{l}$ such that

$$
f(u)=u \cdot i_{f}, g(u)=u \cdot i_{g} \text { and } h(u)=u \cdot i_{h} \text {, for all } u \in P_{l} \text {. }
$$

The triple $\left(i_{f}, i_{g}, i_{h}\right)$ is denoted by $S\left(\theta, Q_{1}\right)$. If $Q_{2}=Q_{1}$, then the isotopism $\theta$ is said to be a Santilli autotopism of $Q_{l}$.

In [11], there were exposed several properties of the set of partial quasigroups having a Santilli autotopism that fixes at least one of the symbols. An exhaustive study of Santilli autotopisms is, however, currently required. This paper is established as a first contribution in this regard. In Section 2, some new general properties of the set of Santilli isotopisms of (associative) partial quasigroups, partial loops and partial groups are analyzed. In Section 3, a classification of the Santilli autotopisms of groups of order $n \leq 6$ is explicitly given. Remark that, even if the number of quasigroups is known for order up to $11[16,17]$, that of partial quasigroups is only known for order up to four $[18,19]$.

\section{Santilli Autotopisms}

From now on, every partial quasigroup of order $\mathrm{n}$ is considered to be formed by the set of elements $\{1, \ldots, n\}$. The set of isotopisms of partial quasigroups of order $\mathrm{n}$ is then denoted as $I_{n}=S_{n} \times S_{n} \times S_{n}$, where $S_{n}$ is the symmetric group on $\{1, \ldots, n\}$. The set of fixed symbols in a permutation $\pi \in \mathrm{S}_{\mathrm{n}}$ is denoted as

$$
\operatorname{Fix}(\pi)=\{u \in\{1, \ldots n\} \text { such that } \pi(u)=u\} .
$$

Let $\theta \in I_{n}$ and let $S Q(\theta), S L(\theta), S A Q(\theta)$ and $S G(\theta)$ be, respectively, the sets of partial quasigroups, partial loops, associative partial quasigroups and partial groups that have $\theta$ as a Santilli autotopism. The next results are satisfied.

Lemma 2.1. Let $\theta=(f, g, h) \in I_{n}$ and $(Q, \cdot) \in S Q(\theta)$ be such that $S(\theta, Q)=\left(i_{f}, i_{g}, i_{h}\right)$. Then, $i_{h}=g\left(i_{f}\right)$. As a consequence,

$$
\left(i \cdot i_{f}\right) \cdot\left(j \cdot i_{g}\right)=(i \cdot j) \cdot\left(i_{f} \cdot i_{g}\right) \text {, for all } i, j \in Q \text {. }
$$

Proof. Given $v \in Q$, let $u \in Q$ be such that $f(u)=v$. Then, $v \cdot i_{h}=h(v)=h(f(u))=h\left(u \cdot i_{f}\right)=f(u) \cdot g\left(i_{f}\right)=v \cdot g\left(i_{f}\right)$ and the result holds from the fact that $Q$ is a partial quasigroup and $h(v)$ $\in Q$.

Proposition 2.2. Let $\theta=(f, g, h) \in I_{n}$ and $(Q, \cdot) \in S Q(\theta)$ be such that $S(\theta, Q)=\left(i_{f}, i_{g}, i_{h}\right)$. If $h=f$, then $i_{f} \in \operatorname{Fix}(g)$.

Proof. The result follows straightforward from Lemma 2.1 and the fact of being $h=f$. 
Lemma 2.3. Let $\theta=(f, g, h) \in I_{n}$. If there exist two permutations $\alpha, \beta \in\{f, g, h\}$ such that $\alpha\left(u_{0}\right)=\beta\left(u_{0}\right)$ for some $u_{0}$ $\in Q$, then $\alpha=\beta$.

Proof. Let $(Q, \cdot)$ be a partial quasigroup in $S Q(\theta)$ and let $i_{\alpha}$, $i_{\beta} \in Q$ be such that $\alpha(u)=u \cdot i_{\alpha}$ and $\beta(u)=u \cdot i_{\beta}$ for all $u \in Q$. Particularly, $u_{0} \cdot i_{\alpha}=\alpha\left(u_{0}\right)=\beta\left(u_{0}\right)=u_{0} \cdot i_{\beta}$. This product is not undefined because $\alpha\left(u_{0}\right) \in Q$. Since $Q$ is a partial quasigroup, it must be then $i_{\alpha}=i_{\beta}$ and hence, $\alpha=\beta$.

Proposition 2.4. Let $\theta=(f, g, h) \in I_{n}$ be such that Fix $(g)=$ $\varnothing$. Then, $f(u) \neq h(u)$ for all $u \in Q$.

Proof. Let $u \in Q$ be such that $f(u)=h(u)$. From Lemma 2.3 it must be $f=h$. Thus, from Lemma 2.1, it is $i_{f}=i_{h}=g\left(i_{f}\right)$ and hence, $i_{f} \in \operatorname{Fix}(g)$, which is a contradiction.

Lemma 2.5. Let $\theta=(f, g, h) \in I_{n}$ and $(Q, \cdot) \in S Q(\theta)$ be such that $S(\theta, Q)=\left(i_{f}, i_{g}, i_{h}\right)$. If there exists $u_{0} \in Q$ such that $h^{m}\left(g\left(u_{0}\right)\right)=g\left(f^{m}\left(u_{0}\right)\right)$ for some positive integer $\mathrm{m}$, then $\mathrm{i}_{\mathrm{g}} \in$ Fix $\left(g^{m}\right)$. As a consequence, if $F i x\left(g^{m}\right)=\varnothing$ for some positive integer $\mathrm{m}$, then $h^{m}(g(u)) \neq g\left(f^{m}(u)\right)$, for all $u \in Q$.

Proof. Let $\mathrm{m}$ be such that $\mathrm{h}^{\mathrm{m}}\left(\mathrm{g}\left(\mathrm{u}_{0}\right)\right)=\mathrm{g}\left(\mathrm{f}^{\mathrm{m}}\left(\mathrm{u}_{0}\right)\right)$ for some $\mathrm{u}_{0}$ $\in$ Q. It is then $\mathrm{f}^{\mathrm{m}}\left(\mathrm{u}_{0}\right) \cdot \mathrm{g}^{\mathrm{m}}\left(\mathrm{i}_{\mathrm{g}}\right)=\mathrm{h}^{\mathrm{m}}\left(\mathrm{u}_{0} \cdot \mathrm{i}_{\mathrm{g}}\right)=\mathrm{h}^{\mathrm{m}}\left(\mathrm{g}\left(\mathrm{u}_{0}\right)\right)=$ $\mathrm{g}\left(\mathrm{f}^{\mathrm{m}}\left(\mathrm{u}_{0}\right)\right)=\mathrm{f}^{\mathrm{m}}\left(\mathrm{u}_{0}\right) \cdot \mathrm{i}_{\mathrm{g}}$. This product is not undefined because $\mathrm{h}^{\mathrm{m}}\left(\mathrm{g}\left(\mathrm{u}_{0}\right)\right) \in \mathrm{Q}$. Since $\mathrm{Q}$ is a partial quasigroup, it must be then $\mathrm{i}_{\mathrm{g}} \in \operatorname{Fix}\left(\mathrm{g}^{\mathrm{m}}\right)$. The consequence is immediate.

Lemma 2.6. Let $\theta=(f, g, h) \in I_{n}$ be such that $|F i x(f)| \cdot|F i x(g)| \cdot|F i x(h)|>0$. Let $(Q, \cdot) \in S Q(\theta)$ be such that $S(\theta, Q)=\left(i_{f}, i_{g}, i_{h}\right)$. If there exist $u_{0} \in \operatorname{Fix}(f), w_{0} \in \operatorname{Fix}(h)$ and $\alpha$ $\in\{f, g, h\}$ such that $\alpha\left(\mathrm{u}_{0}\right)=\mathrm{w}_{0}$, then $i_{\alpha} \in F i x(g)$. Further, if $i_{g} \in$ Fix $(g)$, then $g(u) \in F i x(h)$ for all $u \in F i x(f)$.

Proof. It is satisfied that $u_{0} \cdot \mathrm{i}_{\alpha}=\alpha\left(\mathrm{u}_{0}\right)=\mathrm{w}_{0}=\mathrm{h}\left(\mathrm{w}_{0}\right)=$ $h\left(u_{0} \cdot i_{\alpha}\right)=f\left(u_{0}\right) \cdot g\left(i_{\alpha}\right)=u_{0} \cdot g\left(i_{\alpha}\right)$. Since $w_{0} \in Q$ and $Q$ is a quasigroup, it must be $i_{\alpha} \in$ Fix $(g)$. Let us suppose now that $i_{g}$ $\in F i x(g)$ and let us consider an element $u \in F i x(f)$. Then $g(u)$ $=u \cdot i_{g}=f(u) \cdot g\left(i_{g}\right)=h\left(u \cdot i_{g}\right)=h(g(u))$ and hence, $g(u) \in$ $\operatorname{Fix}(h)$.

The next three results deal with the set of partial loops $S L(\theta)$ having a Santilli isotopism $\theta$ in their autotopism group.

Proposition 2.7. Let $\theta=(f, g, h) \in I_{n}$ and $(Q, \cdot) \in S L(\theta)$ be a partial loop with unit element e. Then, $S(\theta, Q)=(f(e), g(e)$, $g(f(e)))$.

Proof. Let $S(\theta, Q)=\left(i_{f}, i_{g}, i_{h}\right)$. The result follows straightforward from Lemma 2.1 and the fact that $\pi(e) \in Q$. Hence, $\pi(e)=e \cdot i_{\pi}=i_{\pi}$, for all $\pi \in\{f, g\}$.

Lemma 2.8. Let $\theta=(f, g, h) \in I_{n}$. If there exists a permutation $\pi \in\{f, g, h\}$ such that $\operatorname{Fix}(\pi) \neq \varnothing$, then $\pi=I d$.

Proof. Let $(Q, \cdot) \in S L(\theta)$ and $S(\theta, Q)=\left(i_{f}, i_{g}, i_{h}\right)$. Let $\pi \in\{f$, $g, h\}$ and $u_{0} \in Q$ be such that $\pi\left(u_{0}\right)=u_{0}$. Since $u_{0}=u_{0} \cdot i_{\pi}$, the element $\mathrm{i}_{\pi}$ is the unit element of the partial loop. Let $u \in Q$. Since $\pi(u) \in Q$, it is $\pi(u)=u \cdot i_{\pi}=u$ and hence, $\pi=I d$.

Lemma 2.9. Let $\theta=(f, g, h) \in I_{n}$ and $(Q, \cdot) \in S L(\theta)$ be a partial loop with unit element $e$. If $e \in F i x\left(f^{m}\right)$ for some positive integer $\mathrm{m}$, then $h^{m}=g^{m}$. Similarly, if e $\in \operatorname{Fix}\left(\mathrm{g}^{\mathrm{m}}\right)$, then $h^{m}=f^{m}$.
Proof. Let us suppose that $e \in F i x\left(f^{m}\right)$ for some positive integer m. Let $u \in Q$. It is $g^{m}(u)=e \cdot g^{m}(u)=f^{m}(e) \cdot g^{m}(u)=$ $h^{m}(e \cdot u)$. Since $g^{m}(u) \in Q$, it must be $e \cdot u=u$ and hence, $g^{m}(u)$ $=h^{m}(u)$. The last assertion follows analogously.

We focus now on the set $S A Q(\theta)$ of associative partial quasigroups having a Santilli autotopism in their autotopism group.

Proposition 2.10. Let $\theta=(f, g, h) \in \mathrm{I}_{\mathrm{n}}$. If $S A Q(\theta) \neq \varnothing$, then $h=g^{o} f$.

Proof. Let $(Q, \cdot) \in S A Q(\theta)$ and $S(\theta, Q)=\left(i_{f}, i_{g}, i_{h}\right)$. From Lemma 2.1, we know that $i_{h}=g\left(i_{f}\right)$. Hence, for all $u \in Q$, it is verified that $h(u)=u \cdot i_{h}=u \cdot g\left(i_{f}\right)=u \cdot\left(i_{f} \cdot i_{g}\right)=\left(u \cdot i_{f}\right) \cdot i_{g}$ $=g(f(u))$.

Lemma 2.11. Let $\theta=(f, g, h) \in I_{n}$ be such that $S A Q(\theta) \neq \varnothing$ and let $m \leq n$ be a positive integer. Then

a) $S A Q(\theta) \subseteq S A Q\left(\theta^{n}\right)$.

b) $\operatorname{SAQ}(\Theta)=\operatorname{SAQ}\left(\left(\mathrm{f}, \mathrm{g} \circ \mathrm{f}^{\mathrm{m}}, \mathrm{h} \circ \mathrm{f}^{\mathrm{m}}\right)\right)$.

Proof. Let $(Q, \cdot) \in S A Q(\theta)$ be such that $S(\theta, Q)=\left(i_{f}, i_{g}, i_{h}\right)$ and let $m \leq n$ be a positive integer. Then

1. The isotopism $\theta^{n}$ is an autotopism of $(Q, \cdot)$ because $h^{m}(u \cdot v)=h^{m-1}(f(u) \cdot g(v))=\ldots=f^{m}(u) \cdot g^{m}(v)$, for all $u$, $v \in Q$. Since the quasigroup $(Q, \cdot)$ is associative, this is indeed a Santilli autotopism for which $S\left(\theta^{n}, Q\right)=\left(i_{f}{ }^{m}, i_{g}{ }^{m}\right.$, $\left.i_{h}{ }^{m}\right)$.

2. The isotopism $\left(f, g \circ f^{m}, h \circ f^{m}\right)$ is an autotopism of $(Q, \cdot)$ because $h\left(f^{m}(u \cdot v)\right)=h\left((u \cdot v) \cdot i_{f}^{m}\right)=h\left(u \cdot\left(v \cdot i_{f}^{m}\right)\right)=$ $h\left(u \cdot f^{m}(v)\right)=f(u) \cdot g\left(f^{m}(v)\right)$, for all $u, v \in Q$. Since the quasigroup $(Q, \cdot)$ is associative, this is indeed a Santilli autotopism for which $S\left(\left(f, g \circ f^{m}, h \circ f^{m}\right), Q\right)=\left(i_{f}^{m}, i_{f}^{m} \cdot i_{g}\right.$, $\left.i_{f}^{m} \cdot i_{h}\right)$. Hence, $S A Q(\theta) \subseteq S A Q\left(\left(f, g \circ f^{m}, h \circ f^{m}\right)\right)$.

Let us consider now an associative partial quasigroup $\left(Q^{\prime}, *\right) \in S A Q\left(\left(f, g^{o} f^{m}, h^{o} f^{m}\right)\right)$ such that $S\left(\left(f, g^{o} f^{m}, h^{o} f^{m}\right), Q^{\prime}\right)=$ $\left(i_{1}, i_{2}, i_{3}\right)$. It is then verified that $\theta$ is a Santilli autotopism of $(Q, *)$ because, since $f^{n}=I d$, it is $h(u * v)=h\left(f^{n}(u * v)\right)=$ $h\left(f^{m}\left(f^{n-m}(u * v)\right)=h\left(f^{m}\left(u * f^{n-m}(v)\right)=f(u) * g\left(f^{m n}\left(f^{n-m}(v)\right)=f(u) *\right.\right.\right.$ $g\left(f^{n}(v)\right)=f(u) * g(v)$, for all $u, v \in Q^{\prime}$. Further, $S\left(\theta, Q^{\prime}\right)=\left(i_{l}\right.$, $\left.i_{2} *_{i_{1}}{ }^{n-m}, i_{3} *_{i_{1}}{ }^{n-m}\right)$. Hence, $S A Q\left(\left(f, g^{o} f^{m}, h^{o} f^{m}\right)\right) \subseteq S A Q(\theta)$.

In general, given a positive integer $m \leq n$, it is not true that $\operatorname{SAQ}\left(\boldsymbol{\theta}^{n}\right) \subseteq \operatorname{SAQ}(\theta)$. Thus, for instance, the isotopism $\theta=$ ((1234), (1234), (13)(24)) is a Santilli autotopism of the associative quasigroup whose multiplication table is the Latin square exposed in Figure 1. Nevertheless, even if the isotopism $\theta^{2}=((13)(24),(13)(24), I d)$ is a Santilli autotopism of the associative partial quasigroup whose multiplication table is exposed in Figure 3, this is not contained in $\operatorname{SAQ}(\theta)$.

\begin{tabular}{|c|c|c|c|}
\hline & 3 & & 1 \\
\hline & 4 & & 2 \\
\hline & 1 & & 3 \\
\hline & 2 & & 4 \\
\hline
\end{tabular}

Figure 3. Partial Latin square of order 4

Let us finish with a result about the set $S G(\theta)$ of partial groups having a Santilli isotopism in their autotopism group. 
Theorem 2.12. Let $\theta=(f, g, h) \in I_{n}$. If $S G(\theta) \neq \varnothing$ and $F i x(f) \neq \varnothing$, then $g=h$ and $f=I d$.

Proof. The result follows straightforward from Lemma 2.8 and Proposition 2.10.

\section{Santilli Autotopisms of Partial Groups of Order $n \leq 4$}

The results that have been exposed in Section 2 can be taken into account in order to determine explicitly the set of Santilli isotopisms that are autotopisms of partial groups of a given order. To this end, we say that two isotopisms $\theta_{1}=\left(f_{1}, g_{l}, h_{l}\right)$ and $\theta_{2}=\left(f_{2}, g_{2}, h_{2}\right)$ in $I_{n}$ are equivalent if $f_{2}=f_{1}$ and there exists a positive integer $m \leq n$ such that $g_{2}=g_{1}{ }^{o} f_{1}^{m}$ and $h_{2}=h_{1}{ }^{o} f_{l}^{m}$. From assertion (b) in Lemma 2.11, it is verified that $\operatorname{SAQ}\left(\theta_{I}\right)$ $=S A Q\left(\Theta_{2}\right)$. To be equivalent is then an equivalence relation in the set $I_{n}$. Let $[\theta]$ denote the equivalence class of $\theta \in I_{n}$. We expose in Table 1 these equivalence classes for Santilli autotopisms of partial groups of order $n \leq 4$. We focus on the case of non-trivial isotopisms, that is, those that do not coincide with $(I d, I d, I d)$.

Table 1. Santilli autotopisms of partial groups.

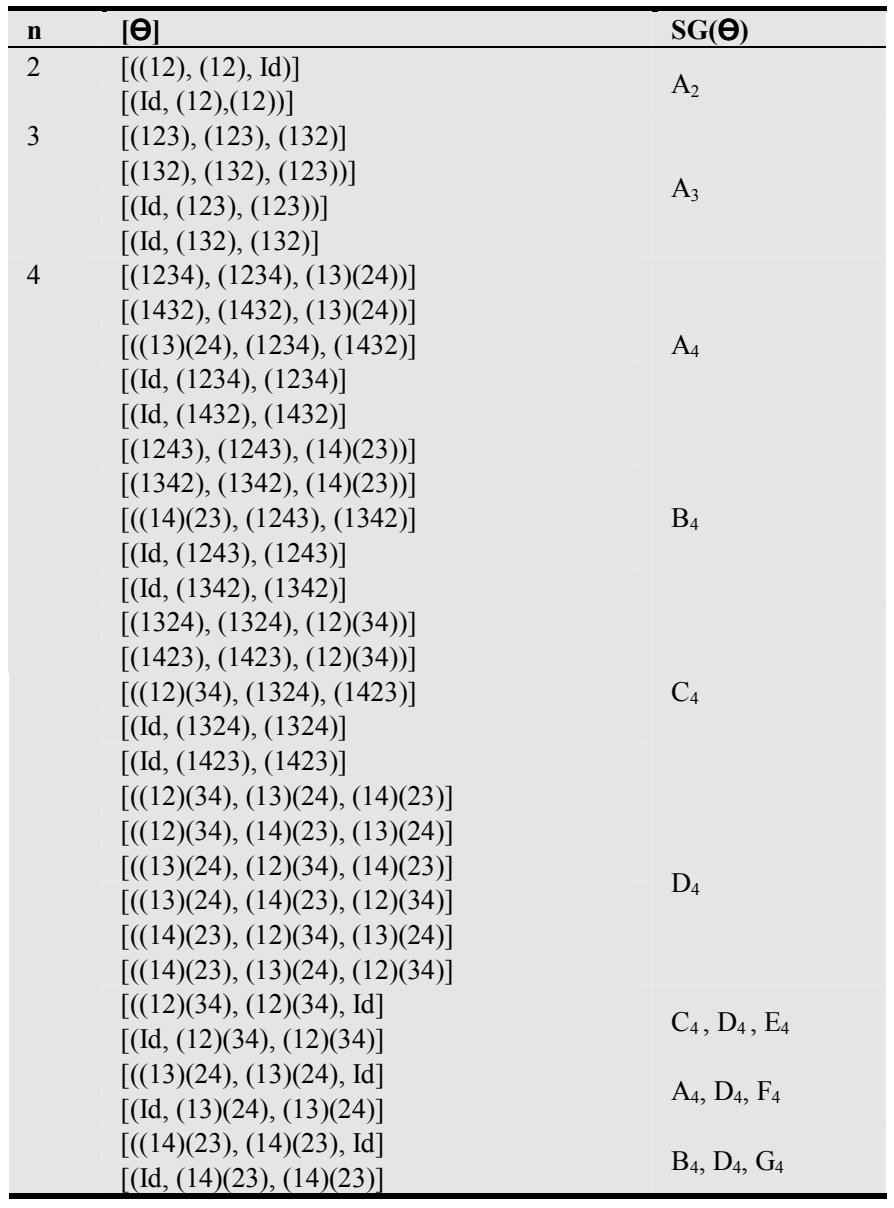

We indicate for each class $[\theta]$ in Table 1 the set $S G(\theta)$ of partial groups that have all the isotopisms of the class in their corresponding autotopism group. The multiplication tables of the elements of these sets are described in Figures 4-12.

\begin{tabular}{|l|l|l|l|}
\hline 1 & 2 & 2 & 1 \\
\hline 2 & 1 & 1 & 2 \\
\hline
\end{tabular}

Figure 4. Partial Latin squares related to $A_{2}$.

\begin{tabular}{|l|l|l|l|l|l|l|l|l|}
\hline 1 & 2 & 3 & 3 & 1 & 2 & 2 & 3 & 1 \\
\hline 2 & 3 & 1 & 1 & 2 & 3 & 3 & 1 & 2 \\
\hline 3 & 1 & 2 & 2 & 3 & 1 & 1 & 2 & 3 \\
\hline
\end{tabular}

Figure 5. Partial Latin squares related to $A_{3}$.

\begin{tabular}{|l|l|l|l|l|l|l|l|l|l|l|l|l|l|l|l|}
\hline 1 & 2 & 3 & 4 & 4 & 1 & 2 & 3 & 3 & 4 & 1 & 2 & 2 & 3 & 4 & 1 \\
\hline 2 & 3 & 4 & 1 & 1 & 2 & 3 & 4 & 4 & 1 & 2 & 3 & 3 & 4 & 1 & 2 \\
\hline 3 & 4 & 1 & 2 & 2 & 3 & 4 & 1 & 1 & 2 & 3 & 4 & 4 & 1 & 2 & 3 \\
\hline 4 & 1 & 2 & 3 & 3 & 4 & 1 & 2 & 2 & 3 & 4 & 1 & 1 & 2 & 3 & 4 \\
\hline
\end{tabular}

Figure 6. Partial Latin squares related to $A_{4}$.

\begin{tabular}{|l|l|l|l|l|l|l|l|l|l|l|l|l|l|l|l|}
\hline 1 & 2 & 3 & 4 & 3 & 1 & 4 & 2 & 2 & 4 & 1 & 3 & 4 & 3 & 2 & 1 \\
\hline 2 & 4 & 1 & 3 & 1 & 2 & 3 & 4 & 4 & 3 & 2 & 1 & 3 & 1 & 4 & 2 \\
\hline 3 & 1 & 4 & 2 & 4 & 3 & 2 & 1 & 1 & 2 & 3 & 4 & 2 & 4 & 1 & 3 \\
\hline 4 & 3 & 2 & 1 & 2 & 4 & 1 & 3 & 3 & 1 & 4 & 2 & 1 & 2 & 3 & 4 \\
\hline
\end{tabular}

Figure 7. Partial Latin squares related to $B_{4}$.

\begin{tabular}{|l|l|l|l|l|l|l|l|l|l|l|l|l|l|l|l|}
\hline 1 & 2 & 3 & 4 & 2 & 1 & 4 & 3 & 4 & 3 & 1 & 2 & 3 & 4 & 2 & 1 \\
\hline 2 & 1 & 4 & 3 & 1 & 2 & 3 & 4 & 3 & 4 & 2 & 1 & 4 & 3 & 1 & 2 \\
\hline 3 & 4 & 2 & 1 & 4 & 3 & 1 & 2 & 1 & 2 & 3 & 4 & 2 & 1 & 4 & 3 \\
\hline 4 & 3 & 1 & 2 & 3 & 4 & 2 & 1 & 2 & 1 & 4 & 3 & 1 & 2 & 3 & 4 \\
\hline
\end{tabular}

Figure 8. Partial Latin squares related to $C_{4}$.

\begin{tabular}{|l|l|l|l|l|l|l|l|l|l|l|l|l|l|l|l|}
\hline 1 & 2 & 3 & 4 & 2 & 1 & 4 & 3 & 3 & 4 & 1 & 2 & 4 & 3 & 2 & 1 \\
\hline 2 & 1 & 4 & 3 & 1 & 2 & 3 & 4 & 4 & 3 & 2 & 1 & 3 & 4 & 1 & 2 \\
\hline 3 & 4 & 1 & 2 & 4 & 3 & 2 & 1 & 1 & 2 & 3 & 4 & 2 & 1 & 4 & 3 \\
\hline 4 & 3 & 2 & 1 & 3 & 4 & 1 & 2 & 2 & 1 & 4 & 3 & 1 & 2 & 3 & 4 \\
\hline
\end{tabular}

Figure 9. Partial Latin squares related to $D_{4}$

\begin{tabular}{|l|l|l|l|l|l|l|l|l|l|l|l|l|l|l|l|}
\hline 1 & 2 & & & 2 & 1 & & & & & 1 & 2 & & & 2 & 1 \\
\hline 2 & 1 & & & 1 & 2 & & & & & 2 & 1 & & & 1 & 2 \\
\hline 3 & 4 & & & 4 & 3 & & & & & 3 & 4 & & & 4 & 3 \\
\hline 4 & 3 & & & 3 & 4 & & & & & 4 & 3 & & & 3 & 4 \\
\hline
\end{tabular}

Figure 10. Partial Latin squares related to $E_{4}$.

\begin{tabular}{|l|l|l|l|l|l|l|l|l|l|l|l|l|l|l|l|}
\hline 1 & & 3 & & & 1 & & 3 & 3 & & 1 & & & 3 & & 1 \\
\hline 2 & & 4 & & & 2 & & 4 & 4 & & 2 & & & 4 & & 2 \\
\hline 3 & & 1 & & 3 & & 1 & 1 & & 3 & & & 1 & & 3 \\
\hline 4 & & 2 & & & 4 & & 2 & 2 & & 4 & & & 2 & & 4 \\
\hline
\end{tabular}

Figure 11. Partial Latin squares related to $F_{4}$.

\begin{tabular}{|l|l|l|l|l|l|l|l|l|l|l|l|l|l|l|l|}
\hline 1 & & & 4 & & 1 & 4 & & & 4 & 1 & & 4 & & & 1 \\
\hline 2 & & & 3 & & 2 & 3 & & & 3 & 2 & & 3 & & & 2 \\
\hline 3 & & & 2 & & 3 & 2 & & & 2 & 3 & & 2 & & & 3 \\
\hline 4 & & & 1 & & 4 & 1 & & & 1 & 4 & & 1 & & & 4 \\
\hline
\end{tabular}

Figure 12. Partial Latin squares related to $G_{4}$.

\section{Acknowledgements}

The authors are very grateful to Prof. Santilli for his valuable help in our research on Santilli isotopisms and cordially congratulate him for his $80^{\text {th }}$ birthday. 


\section{References}

[1] A. A. Albert, "Non-associative algebras. I. Fundamental concepts and isotopy," Ann. of Math. 43:2, pp. 685-707, 1942.

[2] R. H. Bruck, "Some results in the theory of linear non-associative algebras," Trans. Amer. Math. Soc. 56, pp. 141-199, 1944.

[3] R. M. Santilli, "On a possible Lie-admissible covering of the Galilei Relativity in Newtonian Mechanics for nonconservative and Galilei noninvariant systems," Hadronic J. 1, pp. 223-423, 1978. Addendum, ibid 1, pp. 1279-1342, 1978.

[4] R. M. Santilli, "Embedding of Lie algebras in Non-Associative Structures," Nuovo Cimento 51, pp. 570-576, 1967.

[5] R. M. Santilli, "Lie-admissible invariant representation of irreversibility for matter and antimatter at the classical and operator levels," Nuovo Cimento B 121, pp. 443-486, 2006.

[6] P. Nikolaidou and T. Vougiouklis, "The Lie-Santilli admissible hyperalgebras of type An, " Ratio Matematica 26, pp. 113-128, 2014.

[7] J. V. Kadeisvili, "An introduction to the Lie-Santilli theory," Acta Applicandae Mathematicae 50, pp. 131-165, 1998.

[8] R. M. Falcón and J. Núñez, "Fundamentos de la isoteoría de Lie-Santilli," International Academic Press, America-Europe-Asia, 2001.

[9] R. M. Falcón, J. Núñez and A. Aversa, "Mathematical foundations of Santilli isotopies," Algebras, Groups and Geometries 32, pp. 135-308, 2015.

[10] R. M. Falcón and J. Núñez, "A particular case of extended isotopisms: Santilli's isotopisms", Hadronic J. 29:3, pp. 285-298, 2006.

[11] R. M. Falcón and J. Núñez, "Partial Latin squares having a Santilli's autotopism in their autotopism groups," J. Dyn. Syst. Geom. Theor. 5:1, pp. 19-32, 2007.

[12] B. A. Hausmann and O. Ore, "Theory of Quasi-Groups," Amer. J. Math. 59:4, pp. 983-1004, 1937.

[13] A. A. Albert, "Quasigroups I," Trans. Am. Math. Soc. 54, pp. 507-519, 1943.

[14] A. A. Albert, "Quasigroups II," Trans. Am. Math. Soc. 55, pp. 401-419, 1944.

[15] R. H. Bruck, "Some results in the theory of quasigroups," Trans. Amer. Math. Soc. 55, pp. 19-52, 1944.

[16] B. D. McKay, A. Meynert, and W. Myrvold, "Small Latin squares, quasigroups, and loops," J. Combin. Des. 15, pp. 98-119, 2007.

[17] A. Hulpke, P. Kaski, and P. R. J. Östergard, "The number of Latin squares of order 11," Math. Comp. 80, pp. 1197-1219, 2011.

[18] R. M. Falcón, "The set of autotopisms of partial Latin squares", Discrete Math. 313: 11, pp. 1150-1161, 2013.

[19] R. M. Falcón, "Enumeration and classification of self-orthogonal partial Latin rectangles by using the polynomial method", European J. Combin. 48, pp. 215-223, 2015. 